\title{
ON THE EMBEDDING OF VECTOR LATTICES IN $F$-RINGS $\left({ }^{1}\right)$
}

\author{
BY \\ BARRON BRAINERD
}

1. Introduction. In this paper the term $F$-space is used to denote a $\sigma$ complete vector lattice with a weak order unit. (Other authors [8] do not require that a vector lattice with a weak order unit be $\sigma$-complete in order to be an $F$-space.) The term $F$-ring [2] denotes a $\sigma$-complete lattice-ordered rings with an identity which is positive and is a weak order unit. From [8] and [3] it follows that an $F$-space $L$ with weak order unit $u$ determines a unique (up to an isomorphism) regular $F$-ring $R(L, u)$ such that $L$ is isomorphically embedded in $R(L, u)$ and $u$ becomes the identity of $R(L, u)$ under the embedding.

In [7] it is shown that an $F$-space $L$ with weak order unit $u$ can be isomorphically embedded in a ring $N(L)$ of operators on $L$. This ring is referred to by Nakano as the ring of dilatators on $L$. (See $[7, \S 43]$ for definition of dilatator.) $\S 2$ is devoted to showing that $N(L)$ and $R(L, u)$ are isomorphic, as well as showing that if $u$ and $v$ are different weak order units of $L$, then $R(L, u) \cong R(L, v)$.

In $\S 3$ certain propositions are proved which follow from the embeddability of an $F$-space in an $F$-ring.

It is known [3] that the class of idempotents of an $F$-ring $R$ forms a $\sigma$-complete Boolean algebra. If this Boolean algebra supports a countably additive measure $\mu$, then the set $U=\left\{f \in R \mid \mu\left(\bigvee_{n=1}^{\infty}(1 \wedge n|f|)\right)=0\right\}$ is a closed ideal of $R$. $U$ is the generalization for $F$-rings of the family of measurable functions which vanish except on a set of measure zero. In $\$ 4$ properties of the quotient $F$-ring $R-U$ are discussed.

Finally in $\$ 5$ we characterize the class of all bounded linear functionals defined on a regular $F$-ring, as well as the class of linear functionals on an $F$-space $L$ with weak order unit $u$ which can be extended to bounded linear functionals on the regular $F$-ring $R(L, u)$ mentioned in the first paragraph of this Introduction.

The notation of [2] and [3] is used here; in particular: $x^{+}=x \vee 0, x^{-}$ $=(-x)^{+},|x|=x^{+}+x^{-}, \quad \bar{e}_{x}=\bigvee_{n=1}^{\infty}(1 \wedge n|x|)$, and $e_{x}=1-\bar{e}_{x}$. If $L$ is an $\mathrm{F}$ space with weak order unit $u$, then $B(L, u)=\{e \in L \mid e \wedge(u-e)=0\}$ is well known [8] to be a $\sigma$-complete Boolean algebra. If $L$ is an $F$-ring and $u$ is the identity of $L$, then $e \wedge(u-e)=0$ if and only if $e^{2}=e$, and hence in this case

Received by the editors September 7, 1958.

(1) This paper was prepared while the author was a Fellow of the Summer Research Institute of the Canadian Mathematical Congress. 
$B(L, u)$ is the idempotent algebra [3] of $L$. An [1] $l$-ideal $J$ of an $F$-space $L$ is said to be closed if $a_{n} \in J$ for $n \geqq 1$ and $\bigvee_{n-1}^{\infty} a_{n} \in L$ imply $\bigvee_{n=1}^{\infty} a_{n} \in J$. A ring ideal $J$ of an $F$-ring $R$ is said to be closed if it is an $l$-ideal which is closed. An $F$-ring $R$ is regular if for each $a \in R$ there is an $x$ such that $a x a=a$. It is easy to verify that for regular $F$-rings and bounded $F$-rings (those for which the identity 1 is a strong unit [1]) all ring ideals are also $l$-ideals. The converse here is not valid. Indeed if $L$ is an $F$-space with weak order unit $u$ and if $R(L, u)$ is a proper extension of $L$ it can be shown (Theorem 3.1) that $L$ is an $l$-ideal of $R(L, u)$ which need not be a ring ideal. In $\S 3$ it is shown (Theorem 3.2) that a closed $l$-ideal of an $F$-ring is a ring ideal.

A maximal $l$-ideal (ring ideal) $N$ of an $F$-space $L(F$-ring $R$ ) is real if the quotient space $L-N$ (quotient ring $R-N$ ) is isomorphic to the ordered group (ring) of real numbers. An ideal of a Boolean algebra is said to be closed if it is closed with respect to countable sup's. A set $\left\{e_{\gamma} \mid \gamma \in \Gamma\right\}$ of nonnegative elements of an $F$-space is said to be orthogonal if $e_{\gamma_{1}} \wedge e_{\gamma_{2}}=0$ whenever $\gamma_{1} \neq \gamma_{2}$. An $F$-space $L$ is orthogonally complete $[7$, p. 156] if for each orthogonal sequence $\left\{f_{n}\right\}$ of non-negative elements of $L$ the supremum $\bigvee_{n=1}^{\infty} f_{n}$ belongs to $L$. If $e$ is an element of a Boolean algebra then $\bar{e}$ denotes the complement of $e$.

A linear functional $\xi$ on an $F$-space $L$ is said to be continuous if for each nonincreasing sequence $\left\{a_{n}\right\}$ of elements of $L$ such that $\bigwedge_{n=1}^{\infty} a_{n}=0$ we have $\lim _{n} \xi\left(a_{n}\right)=0$. If $B$ is a $\sigma$-complete Boolean algebra, by a measure on $B$ we mean a functional $\mu$ on $B$ which satisfies the following conditions: (i) if $e_{1}, e_{2} \in B$ and $e_{1} \wedge e_{2}=0$, then $\mu\left(e_{1}\right)+\mu\left(e_{2}\right)=\mu\left(e_{1} \bigvee e_{2}\right)$, (ii) $\mu(e) \geqq 0$ for all $e \in B$. A measure $\mu$ on $B$ is countably additive if when $\left\{e_{n}\right\}$ is an orthogonal sequence of elements of $B$, then $\mu\left(\bigvee_{n=1}^{\infty} e_{n}\right)=\sum_{n=1}^{\infty} \mu\left(e_{n}\right)$. A measure $\mu$ on $B$ is normal if $\mu(1)=1$. It is clear that any nontrivial measure $\mu$ on $B$ can be normalized by dividing by $\mu(1)$.

2. Extensions of $F$-spaces. Let $L$ be an $F$-space with weak order unit $u$. In [8] a lattice-ordered ring $R(L, u)$ is constructed such that $L$ is isomorphic to an $F$-subspace of $R(L, u)$ and the image of $u$ under this isomorphism is the identity of $R(L, u)$. In [3] we show that $R(L, u)$ is a regular $F$-ring, and then it is clear from the results of $[3 ; 8]$ that $B(L, u)$ is mapped onto the idempotent algebra of $R(L, u)$ by this embedding isomorphism. We also show in [3] that the mapping which carries a regular $F$-ring $R$ with identity 1 into its idempotent algebra $B(R, 1)$ is a mapping which is one to one (up to an isomorphism) from the set of regular $F$-rings onto the set of $\sigma$-complete Boolean algebras. In addition we show that every regular $F$-ring can be faithfully represented as the $\sigma$-homomorphic image of an $M$-ring [2]. The " $\sigma$ " preceding the word "homomorphism" indicates that this homomorphism preserves countable sup's and inf's.

Nakano considers another method of extending an $F$-space. He defines $[7, \S 33]$ what he calls a completion of a $\sigma$-complete vector lattice. This com- 
pletion is an extension of the vector lattice which is unique up to an isomorphism. If the vector lattice is an $F$-space $L$, then the completion $N(L)$ of $L$ is isomorphic to the dilatator ring of $L$. This result is embodied in [7, Theorem 45.7]. The dilatators of $L$ are discussed in $[7, \S \S 44,45]$. In order to prove that $N(L)$ and $R(L, u)$ are isomorphic, we first consider the following lemma.

Lemma 2.1. If $L$ is an $F$-space with weak order units $u$ and $v$, then $B(L, u)$ and $B(L, v)$ are isomorphic Boolean algebras and therefore the F-rings $R(L, u)$ and $R(L, v)$ are isomorphic.

Proof. Consider $R(L, u)$ and identify $L$ with its isomorphic copy in $R(L, u)$. For the remainder of this proof all elements considered are considered as elements of $R(L, u)$. In particular any products discussed are products as defined in $R(L, u)$. From [8] it follows that $B(R(L, u), u)=B(L, u)$, and $B(L, v)$ is a subset of $R(L, u)$.

The mapping $e \rightarrow v e$ is an isomorphisn of $B(L, u)$ onto $B(L, v)$. Indeed, if $e \in B(L, u)$, then $v e \wedge(v-v e)=v[e \wedge(u-e)]=0$ and hence $v e \in B(L, v)$. Since $v$ is a weak order unit of $L$ it is a weak order unit of $R(L, u)$ and hence $v^{-1} \in R(L, u)$. The first clause of the preceding sentence follows because every element of $R(L, u)$ is a sup of disjoint elements of $L$ and the second clause follows from regularity together with the statement (valid in $R(L, u)$ ): If $a \geqq 0, b \geqq 0$, then $a \wedge b=0 \Leftrightarrow a b=0$.

The existence of $v^{-1} \in R(L, u)$ ensures that every element of $B(L, v)$ is the image of a unique element of $B(L, u)$ and hence the mapping $e \rightarrow v e$ is one-to-one and onto. The lattice operations are preserved because of the distributivity of multiplication with respect to the lattice operations and because the complement of $e \in B(L, v)$ is of the form $v-e$.

That $R(L, u) \cong R(L, v)$ follows from the results of [3].

THEOREM 2.2. If $L$ is an $F$-space with weak order unit $u$, the dilatator ring $N(L)$ of $L$ is an F-ring which is isomorphic to $R(L, u)$.

Proof. Since $R(L, u)$ satisfies conditions (1) through (4) in $[7, \S 33]$ it follows that $R(L, u)$ is a completion of $L$. Moreover the $F$-spaces $R(L, u)$ and $N(L)$ are isomorphic because $N(L)$ is a completion and [7, Theorem 33.4] all completions of $L$ are isomorphic. Let $\rho$ be the isomorphism of $R(L, u)$ onto $N(L)$. Now $\rho(u)$ is a weak order unit of $N(L)$, and $B(N(L), \rho(u))$ $\cong B(R(L, u), u) \cong B(L, u)$. It follows from [3, Theorem 7] and from the remark in the first paragraph of this section that the $F$-rings $R(N(L), \rho(u))$ and $R(L, u)$ are isomorphic.

From [7, Theorems 29.9 and 44.1] we deduce that the dilatator ring $N(L)$ of $L$ possesses a multiplicative identity $1 \geqq 0$ which is a weak order unit. Therefore the dilatator ring $N(L)$ is an $F$-ring. Now (Lemma 2.1) $F$-rings $R(N(L), \rho(u))$ and $R(N(L), 1)$ are isomorphic. From [7, Theorem 44.1] and 
the properties of Nakano's proper spaces, it follows that every principal ideal of $N(L)$ is generated by an idempotent. Hence $N(L)$ is regular and the $F$-rings $N(L)$ and $R(N(L), 1)$ are isomorphic. Thus

$$
R(L, u) \cong R(N(L), \rho(u)) \cong R(N(L), 1) \cong N(L) .
$$

CoROllaRy 2.3. A necessary and sufficient condition for an $F$-space $L$ to be orthogonally complete is that it be possible to define a product in $L$ which turns $L$ into a regular F-ring.

Proof. If $L$ is orthogonally complete, then by the definition of completion $[7, \S 33]$ it is clear that the identity mapping on $L$ is a completion of $L$. Since [7, Theorem 33.4] the completion of $L$ is unique, it follows that $L$ is its own dilatator ring. Therefore a product may be defined in $L$ with respect to which $L$ forms a regular $F$-ring.

Conversely, if $R$ is a regular $F$-ring, $R \cong R(R, 1)$ and Theorem 2.2 implies $R \cong N(R)$ and hence $R$ is orthogonally complete.

3. Some properties of $F$-spaces. This section is devoted to the study of some properties of $F$-spaces which follow from their embeddability in $F$-rings.

Let $L$ stand for an $F$-space with weak order unit 1 . Since $L$ is an $F$ subspace of $R(L, 1)$, certain pairs $a, b$ of elements of $L$ have the property that their product $a b$ in $R(L, 1)$ also belongs to $L$. Again we identify $L$ with its isomorphic copy in $R(L, 1)$.

TheOREm 3.1. The $F$-space $L$ is an $l$-ideal of $R(L, 1)$, and if $a, b \in L$ and $|a| \leqq \lambda \cdot 1$ for some $\lambda>0$, then $a b \in L$.

Proof. $L$ is clearly a subspace of $R(L, 1)$; in order to show that it is an $l$-ideal we must prove that $a \in R(L, 1), b \in L$, and $0 \leqq a \leqq|b|$ imply $a \in L$. The element $a \in R(L, 1)$ is the sup of a sequence of elements of $L[1$, p. 251], and since $L$ is $\sigma$-complete, $a \in L$. Therefore $L$ is an $l$-ideal of $R(L, 1)$.

To prove the second part of the theorem, note that $\lambda|b| \in L$ and $|a b|$ $\leqq \lambda|b|$. Hence $a b \in L$ because $L$ is an $l$-ideal of $R(L, 1)$.

Theorem 3.2. Let $M$ be a closed l-ideal of $L$ and let $b \in M$. If $a \in L$ and $a b \in L$, then $a b \in M$; hence closed l-ideals of F-rings are ring ideals.

Proof. Assume $a \geqq 0$ and $b \geqq 0$. Then for each integer $n \geqq 0$,

$$
a_{n}=a\left[e_{(a-n)^{+}}-e_{(a-n+1)^{+}}\right] b \leqq n b .
$$

Since $a_{n}$ is dominated by an element $n b \in M, a_{n} \in M$ for all $n \geqq 0$. Therefore $a b=\bigvee_{n=1}^{\infty} a_{n} b$ belongs to $M$.

If $a$ and $b$ are not non-negative, then $a b \in L$ implies $|a b| \in L$. Since $a^{+} b^{+}, a^{-} b^{-}, a^{+} b^{-}$, and $a^{-} b^{+}$are all dominated by $|a b| \in L$, it follows that each belongs to $L$ and therefore each belongs to $M$.

THEOREM 3.3. The correspondence $\phi: M \rightarrow M \cap L$ is a one-to-one mapping of the closed maximal ideals of $R(L, 1)$ onto the closed maximal l-ideals of $L$. The 
inverse of $\phi$ can be written $\phi^{-1}: M \rightarrow(M \cap B(L, 1)) R(L, 1)$ where $A R(L, 1)$ stands for the (ring) ideal of $R(L, 1)$ generated by the set $A \subseteq R(L, 1)$.

Proof. For the remainder of this proof we use the notation $R$ for $R(L, 1)$ and $B$ for $B(L, 1)$, and again $L$ is identified with its isomorphic copy in $R$. Let $\Omega$ stand for the class of closed maximal ideals of $R$ and let $\Omega^{\prime}$ stand for the class of closed maximal $l$-ideals of $L$.

In order to show $\phi(\Omega) \subseteq \Omega^{\prime}$ consider $L-M \cap L$ where $M \in \Omega$; this ordered group is isomorphic to the ordered group $[L+M]-M$ because $M$ is an $l$-ideal. In $[2$, p. 677] it is shown that $\bar{R}+M=R$ where $\bar{R}$ signifies the $F$-ring composed of those elements $b \in R$ for which a $\lambda>0$ exists such that $|b|$ $\leqq \lambda \cdot 1$. Since $L \supseteq \bar{R}$, it follows that $L+M=R$ and $[L+M]-M$ is an ordered group [2, Theorem 5] which is isomorphic to $G$, the ordered group of real numbers. Therefore $L-M \cap L$ and $G$ are ordered group-isomorphic, and since $G$ has no proper $l$-ideals, $M \cap L \in \Omega^{\prime}$.

To show $\phi(\Omega) \supseteq \Omega^{\prime}$ and $\phi \circ \phi^{-1}$ is the identity mapping on $\Omega^{\prime}$, let $M^{\prime} \in \Omega^{\prime}$. Then $M^{\prime} \cap B$ is a closed maximal ideal of $B$. By [4, Lemma 2] $M=\left(M^{\prime} \cap B\right) R$ is a closed maximal ideal of $R$. Both propositions to be proved are valid if the sets $M \cap L$ and $M^{\prime}$ are equal. Since $R$ is regular, every element $a \in R$ has the property $a \in M$ if and only if $\bar{e}_{a} \in M^{\prime} \cap B$, and because of Theorem 3.2 every element $a \in L$ has the property $a \in M^{\prime}$ if and only if $\bar{e}_{a} \in M^{\prime} \cap B$. From these two statements it is easy to deduce that $M \cap L=M^{\prime}$.

Since it is a trivial consequence of $\left[4\right.$, Lemma 2] that $\phi^{-1}\left(\Omega^{\prime}\right) \subseteq \Omega$, the theorm follows if it can be shown that $\phi^{-1}\left(\Omega^{\prime}\right) \supseteq \Omega$ and $\phi^{-1} \circ \phi$ is the identity mapping on $\Omega$. Let $M \in \Omega$. Then $M \cap L \in \phi(\Omega)$ and $\phi^{-1}(M \cap L)=(M \cap L \cap B) R$ $=(M \cap B) R=M$ by $\left[4\right.$, Lemma 2]. Therefore $\phi^{-1}\left(\Omega^{\prime}\right) \supseteq \Omega, \phi^{-1} \circ \phi(M)=M$ for all $M \in \Omega$, and the theorem follows.

Corollaky 3.4. If $M^{\prime}$ is a closed maximal l-ideal of $L$, then $L-M^{\prime}$ is isomorphic to the ordered group $G$ of real numbers.

Proof. By Theorem 3.3, $M^{\prime}=M \cap L$ where $M$ is a closed maximal ideal of $R(L, 1)$. In the proof of Theorem 3.3 it is shown that $I-M \cap L$ is isomorphic to $G$.

Corollary 3.5. An F-space $L$ is isomorphic to an F-space of measurable functions if and only if the intersection of the class $\Omega^{\prime}$ of all its closed maximal l-ideals is the zero ideal.

Proof. Consider the class $\phi^{-1}\left(\Omega^{\prime}\right)=\Omega$. The set $I=\bigcap_{\phi^{-1}}\left(\Omega^{\prime}\right)$ is an $l$-ideal of $R(L, 1)$. Therefore $a \in I$ implies $|a| \wedge 1 \in I$, but $|a| \wedge 1$ is an element of $L$ and hence $|a| \wedge 1 \in \cap \Omega^{\prime}$. By hypothesis $|a| \wedge 1=0$, and since 1 is a weak order unit of $R(L, 1)$ we have $a=0$. Therefore $\cap \Omega=\{0\}$ and [2, Theorem 7] $R(L, 1)$ is isomorphic to an $F$-ring of measurable functions. The corollary then follows immediately. 
An $F$-space $L$ (Boolean algebra $B$ ) is $m$-complete, where $m$ is a cardinal number larger than or equal to $\aleph_{0}$, provided every subset $A$ of $L_{1}(B)$ which is bounded above by an element of $L(B)$ and has power less than or equal to $m$ has a supremum in $L(B)$.

Theorem 3.6. If $L$ is an $F$-space with weak order unit 1 , then $B(L, 1)$ is an $m$-complete Boolean algebra if and only if $L$ is an m-complete $F$-space.

Proof. If $B(L, 1)$ is $m$-complete, then it can be verified directly from Olmsted's definition of $R(L, 1)$ that $R(L, 1)$ is an $m$-complete $F$-ring. Since $L$ is an $l$-ideal (Theorem 3.1) of $R(L, 1)$ it follows that the supremum of any set $A(|A| \leqq m)$ of elements of $L$ bounded above by an element of $L$ belongs to $L$ and hence $L$ is $m$-complete.

It is clear that the $m$-completeness of $L$ implies the $m$-completeness of $B(L, 1)$.

An $F$-space $L$ (Boolean algebra $B$ ) is complete provided $L(B)$ is $m$-complete for each cardinal number $m$.

CoRollary 3.7. If $L$ is an $F$-space with weak order unit 1 , then $B(L, 1)$ is complete if and only if $K$ is complete.

4. Functionals on $F$-rings and measures on Boolean algebras. Let $L$ be an $F$-space with weak order unit 1 . If $\mu$ is a non-negative (continuous) linear functional on $L$, then the restriction of $\mu$ to $B(L, 1)$ is a (countably additive) measure on $B(L, 1)$. Of course not every (countably additive) measure on $B(L, 1)$ is a restriction of a non-negative (continuous) linear functional on $L$. It is sometimes of interest to consider those $F$-spaces $L$ for which a countably additive measure $\mu$ can be defined on $B(L, 1)$ and to form the quotient space of $L$ by the $l$-ideal $U$ composed of those elements $f \in L$ which are the abstract counterpart of functions nonvanishing on a set of measure zero. Let $U$ $=\left\{g \in L \mid \mu\left(\bar{e}_{o}\right)=0\right\}$. It is clear that if $L$ is an $F$-space of measurable functions, then $U$ is the set of all elements of $L$ which vanish on the complement of a set $A$ such that $\mu\left(x_{A}\right)=0$.

Let $R$ be an $F$-ring and let $B=B(R, 1)$. Assume $\mu$ is a countably additive normal measure on $B$. It is clear that there is no loss in generality in assuming $\mu$ normal. valid:

Theorem 4.1. If $U=\left\{a \in R \mid \mu\left(\bar{e}_{a}\right)=0\right\}$, then the following statements are

(1) $U$ is a closed ideal of $R$.

(2) $R-U$ is a complete regular F-ring.

(3) If $a$ stands for the image of $a \in R$ under the natural homomorphism $R \rightarrow R-U$ and if by definition $\hat{\mu}(\hat{a})=\mu(a)$ where $a \in \hat{a} \cap B$, then $\hat{\mu}$ is a normal positive countably additive measure on $B(R-U, \hat{1})$.

REMARK. A measure $\mu$ on a Boolean algebra $B$ is positive if $\mu(a)=0$ if and only if $a=0$. 
Proof. (1) First we show that $U$ is an $l$-ideal. By definition $a \in U$ if and only if $\bar{e}_{a} \in U$. Therefore since $a \in U, b \in R$ such that $|b| \leqq|a|$ implies $b \in U$. Since for $\alpha \neq 0$ the equation $\bar{e}_{a}=\bar{e}_{\alpha a}$ is valid, $a \in U$ implies $\alpha a \in U$ for all real numbers $\alpha$. Let $f, g \in U$. Then $|f+g| \leqq 2(|f| \vee|g|)$ and hence $\bar{e}_{(f+\theta)}$ $\leqq \bar{e}_{f} \vee \bar{e}_{g}$. Therefore $\mu\left(\bar{e}_{(f+g)}\right)=0$ and $U$ is an $l$-ideal.

To show $U$ is closed let $f_{n} \in U$ for $n \geqq 1$ and let $f=\bigvee_{n=1}^{\infty} f_{n}$ belong to $R$. It is a matter of direct verification to show that $\bigvee_{n=1}^{\infty} \bar{e}_{f_{n}}=\bar{e}_{f}$. Since $\mu\left(\bar{e}_{f}\right)$ $\leqq \sum_{n=1}^{\infty} \mu\left(\bar{e}_{f_{n}}\right)$, it follows that $\mu\left(\bar{e}_{f}\right)=0$ and $f \in U$.

That $U$ is a closed ring ideal of $R$ then follows from Theorem 3.2.

(2) The results of [3, Theorem 2] imply that $R-U$ is a regular $F$-ring. Since the quotient Boolean algebra $B / B \cap U$ and $B(R-U, \hat{1})$ are isomorphic, it follows from [9, Theorem 4.7] that $B(R-U, \hat{1})$ is a complete Boolean algebra. Corollary 3.7 yields the result that $R-U$ is complete.

(3) The functional $\hat{\mu}$ is well defined on $\hat{B}=B(R-U, \hat{\mathbf{1}})$. Indeed for each $\hat{a} \in \hat{B}$ if $a, b \in \hat{a} \cap B$, then $|a-b|=(a-a \wedge b)+(b-a \wedge b) \in U$ and so $a-a \wedge b$ and $b-a \wedge b$ both belong to $U$. Since for each $a \in B$ we have $a=\bar{e}_{a}$, it follows that $\mu(a-a \wedge b)=\mu(b-a \wedge b)=0$. Therefore $\mu(a)=\mu(a \wedge b)=\mu(b)$.

$\hat{\mu}$ is clearly normal, positive, and finitely additive. To prove that $\hat{\mu}$ is countably additive, let $\left\{\hat{e}_{n}\right\}$ be an orthogonal sequence of elements of $\hat{B}$. Since $U$ is a closed ideal of $R$, an orthogonal sequence $\left\{e_{n}\right\}$ can be constructed in $B$ such that $e_{n}$ maps into $\hat{e}_{n}$ under the natural homomorphism of $R$ onto $R-U$. Indeed, let $\left\{b_{n}\right\}$ be a sequence of idempotents of $R$ such that $b_{n} \rightarrow \hat{e}_{n}$ under the natural homomorphism. Let $\psi$ represent this natural homomorphism; then $\psi\left(b_{n}\right)=\hat{e}_{n}$. For each pair $m, n(m \neq n), b_{n} \wedge b_{n}=k_{m n}$ and $k_{m n}=k_{n m}$ belongs to $U \cap B$. Therefore $\psi\left(b_{n} \bar{k}_{m n}\right)=\psi\left(b_{n}\right), \psi\left(b_{m} \bar{k}_{m n}\right)=\psi\left(b_{m}\right)$, and $\left(b_{n} \bar{k}_{m n}\right)$ $\wedge\left(b_{m} \bar{k}_{m n}\right)=0$. Now $k=\mathrm{V}_{n, m} k_{m n}$ belongs to $U$ and $\bar{k} \leqq \bar{k}_{m n}$ for $m \geqq 1, n \geqq 1$. Therefore $\psi\left(b_{n} \bar{k}\right)=\psi\left(b_{n}\right)$ for all $n \geqq 1$ and $b_{n} \bar{k} \wedge b_{m} \bar{k}=0$ if $m \neq n$. Thus if $e_{n}=b_{n} \vec{k},\left\{e_{n}\right\}$ is the required orthogonal sequence.

It is now clear that

$$
\begin{aligned}
\hat{\mu}\left(\bigvee_{n=1}^{\infty} \hat{e}_{n}\right) & =\hat{\mu}\left(\bigvee_{n=1}^{\infty} e_{n}\right) \hat{}=\mu\left(\bigvee_{n=1}^{\infty} e_{n}\right) \\
& =\sum_{n=1}^{\infty} \mu\left(e_{n}\right)=\sum_{n=1}^{\infty} \hat{\mu}\left(\hat{e}_{n}\right),
\end{aligned}
$$

and hence $\hat{\mu}$ is countably additive.

We return to the consideration of non-negative linear functionals on an $F$-space $L$ with weak order unit 1.

THEOREM 4.2. If $\mu$ is a non-negative continuous linear functional on $L$, then

$$
\mu(|f|)=0 \Leftrightarrow \mu\left(\bar{e}_{f}\right)=0 .
$$

Proof. Suppose $\mu(|f|)=0$. Then $\mu(n|f| \wedge 1)=0$. The sequence $\bar{e}_{f}-n|f| \wedge 1$ is a nonincreasing sequence with infimum zero. Therefore $\lim _{n} \mu\left(\bar{e}_{f}-n|f| \wedge 1\right)$ $=\lim _{n}\left[\mu\left(\bar{e}_{f}\right)-\mu(n|f| \wedge 1)\right]=\mu\left(\bar{e}_{f}\right)-\lim _{n} \mu(n|f| \wedge 1)=\mu\left(\bar{e}_{f}\right)=0$. 
Conversely, suppose $\mu\left(\bar{e}_{f}\right)=0$. Then $\mu(n|f| \wedge 1)=0$ for all $n$ and hence $\mu(\alpha|f| \wedge 1)=0$ for all $\alpha \geqq 0$. This then implies that $\mu(|f| \wedge \alpha \cdot 1)=0$ for all $\alpha \geqq 0$. Again $|f|-|f| \wedge n \cdot 1$ is a nonincreasing sequence with infimum zero, so $\lim _{n} \mu(|f|-|f| \wedge n \cdot 1)=0$ and hence $\mu(|f|)=0$.

TheOREM 4.3. If $\mu$ is a non-negative continuous linear functional on $L$, then

(1) $V=\{f \in L \mid \mu(|f|)=0\}$ is a closed l-ideal of $L$.

(2) $L-V$ is a complete F-space.

(3) If $\hat{a} \in L-V$ is the image of $a \in L$ under the natural homomorphism of $L$ onto $L-V$, then if by definition $\hat{\mu}(\hat{a})=\mu(a)$, it follows that $\hat{\mu}$ is a continuous positive linear functional on $L-V$.

Proof. (1) By Theorem 4.2, $V=\left\{f \in R(L, 1) \mid \mu\left(\bar{e}_{f}\right)=0\right\} \cap L$ where $L$ is identified with its isomorphic copy in $R(L, 1)$. Therefore $V$ is a closed $l$-ideal by Theorem 4.1 .

(2) It is easy to verify that $L-V$ is an $F$-space with weak order unit $\hat{\mathbf{1}}$. Since $V \cap B(L, 1)$ is the set of elements of $B(L, 1)$ with measure zero, $B(L, 1) / V \cap B(L, 1)$ is $[9$, Theorem 4.7] a complete Boolean algebra, and since $\hat{B}=B(L-V, \hat{1})$ is isomorphic to $B(L, 1) / V \cap B(L, 1)$, it follows that $\hat{B}$ is complete. Hence $L-V$ is complete (Corollary 3.7).

(3) $\hat{\mu}$ is well defined on $L-V$ because $x, y \in \hat{x}$ implies $|x-y| \in \hat{0}$ and hence $\mu(|x-y|)=0$. Therefore $\mu(x)=\mu(y)$. The functional $\hat{\mu}$ is clearly linear and positive. To show it is continuous let $\left\{\hat{x}_{n}\right\}$ be a nonincreasing sequence of elements in $L-V$ such that $\bigwedge_{n=1}^{\infty} \hat{x}_{n}=0$. A sequence $\left\{x_{n}\right\}$ is contained in $L$ such that $x_{n} \in \hat{x}_{n}$ and $x_{n} \geqq 0$ for all $n \geqq 1$.

Let $y_{n}=\bigwedge_{k=1}^{n} x_{k}$. Then it is clear that $\hat{y}_{n}=\hat{x}_{n}$ and that $y=\bigwedge_{n=1}^{\infty} y_{n}$ belongs to $\hat{0}$. Therefore $\left\{z_{n}\right\}$ where $z_{n}=y_{n}-y$ is a sequence of elements of $L$ such that $z_{n} \geqq 0, z_{n} \geqq z_{n+1}, z_{n} \in \hat{x}_{n}$ for all $n \geqq 1$, and $\bigwedge_{n=1}^{\infty} z_{n}=0$. Now $\lim _{n} \mu\left(z_{n}\right)=0$ and hence $\lim _{n} \hat{\mu}\left(\hat{x}_{n}\right)=0$ and the continuity of $\hat{\mu}$ is established.

REMARK. Part (2) of the above proof can be established directly from part (3). Indeed, the positiveness of $\hat{\mu}$ can be shown to imply that $B$ is complete. Furthermore, if $L$ is an $F$-space with weak order unit 1 and $B(L, 1)$ supports a positive measure, then $L$ and $R(L, 1)$ are complete $[9$, Theorem 4.7 and Corollary 3.7].

5. Bounded linear functionals on regular $F$-rings. A linear functional $\xi$ defined on an $F$-space $L$ is bounded if bounded sets of elements of $L$ are carried by $\xi$ into bounded sets of real numbers. It is well known [7] that every such bounded linear functional is the difference of two non-negative linear functionals.

Let $\mu$ be a nontrivial non-negative linear functional defined on a regular $F$-ring $R$ with identity 1 . The following lemmas are used for the characterization of the class of bounded linear functionals on $R$.

LEмma 5.1. If $\left\{e_{n}\right\}$ is an orthogonal sequence of idempotents of $R$, then $\mu\left(e_{n}\right)=0$ for all $n$ larger than some fixed $n_{0}$. 
Proof. Suppose there is an orthogonal sequence $\left\{e_{n}\right\}$ of idempotents of $R$ such that $\mu\left(e_{n(K)}\right) \neq 0$ for an infinite sub-sequence $\{n(K)\}$ of the natural numbers. Since $R$ is orthogonally complete (Corollary 2.3), the element

$$
g=\bigvee_{K=1}^{\infty} e_{n(K)} / \mu\left(e_{n(K)}\right)
$$

belongs to $R$. Thus we find that $\mu(g) \geqq n$ for all $n \geqq 1$ which contradicis the hypothesis that $\mu$ is defined throughout $R$.

LEMMA 5.2. The restriction of $\mu$ to $B=B(R, 1)$ is a countably additive measure.

Proof. (This proof is essentially due to Mackey [6].) Let $\left\{e_{n}\right\}$ be an orthogonal sequence of idempotents of $R$. Then (Lemma 5.1) $\mu\left(e_{n}\right)=0$ for $n$ larger than some natural number $n_{0}$. Since $\mu\left(\bigvee_{n=1}^{\infty} e_{n}-\sum_{n-1}^{n_{0}} e_{n}\right)=\mu\left(\bigvee_{n-1}^{\infty} e_{n}\right)$ $-\sum_{n=1}^{n_{0}} \mu\left(e_{n}\right)$, it is only necessary to show that

$$
\mu\left(\bigvee_{n=1}^{\infty} e_{n}-\sum_{n=1}^{n} e_{n}\right)=0
$$

thus no generality is lost in considering the case where $\mu\left(e_{n}\right)=0$ for all $n \geqq 1$. The element $g=\bigvee_{n=1}^{\infty} n e_{n}$ exists in $R$ because $R$ is orthogonally complete (Corollary 2.3). Therefore

$$
g-\sum_{k=1}^{m} k e_{k} \geqq m\left(\bigvee_{n=1}^{\infty} e_{n}-\sum_{k=1}^{m} e_{k}\right),
$$

and hence $\mu(g) \geqq m \mu\left(\bigvee_{n=1}^{\infty} e_{n}\right)$ for all $m \geqq 1$. Thus $\mu\left(\bigvee_{n=1}^{\infty} e_{n}\right)=0$, and $\mu$ is countably additive.

Let $U_{\mu}=\left\{a \in R \mid \mu\left(\bar{e}_{a}\right)=0\right\}$. Since $\mu$ is a countably additive measure, $U_{\mu}$ is a closed ideal of $R$ and hence $\hat{R}=R-U_{\mu}$ possesses the properties indicated in Theorem 4.1. Let $\hat{a}$ stand for the image of $a \in R$ under the natural homomorphism of $R$ onto $\hat{R}=R-U_{\mu}$.

LEMMA 5.3. The Boolean algebra $\hat{B}=B(\hat{R}, 1)$ is atomic and the set of all its atoms is finite.

Proof. To show $\hat{B}$ is atomic, first note that ascending (descending) chains of elements of $\hat{B}$ have finite length. This follows from Lemma 5.1 because if $\left\{\hat{e}_{n}\right\}$ is an ascending chain of elements of $\hat{B}$, then $\left\{\hat{e}_{n+1}-\hat{e}_{n}\right\}$ is an orthogonal sequence and if $\hat{\mu}$ is the positive countably additive measure on $\hat{B}$ induced by $\mu$ (see Theorem 4.1), then $\hat{\mu}\left(\hat{e}_{n+1}-\hat{e}_{n}\right)=0$ for $n>n_{0}$. Therefore $\hat{e}_{n_{0}+1}$ $=\hat{e}_{n_{0}+2}=\cdots$ and the chain $\left\{\hat{e}_{n}\right\}$ has finite length. A similar proof can be given for descending chains.

Since descending chains have finite length, it follows that every element covers an atom of $\hat{B}$ and hence $\hat{B}$ is atomic. Similarly since ascending chains have finite length there can be at most a finite set of atoms in $\hat{R}$. 
Now it is possible to characterize the bounded linear functionals on $R$.

THEOREM 5.4. Every nontrivial bounded linear functional on $R$ is a finite linear combination of ring-homomorphisms of $R$ onto the real field.

Proof. Since every bounded linear functional on $R$ is the difference of two non-negative linear functionals, the theorem will follow in general if it can be proved for non-negative linear functionals. Let $\mu$ be a nontrivial non-negative linear functional. From Lemma 5.2 it follows that $\mu$ is a countably additive measure on $B(R, 1)$. If we adopt the conventions stated in the paragraph preceding Lemma 5.3, then it is clear from Lemma 5.3 that $\hat{R}=R-U_{\mu}$ is the $F$-ring of ordered $n$-tupples of real numbers for some fixed $n$ and that the atoms $\hat{a}_{1}, \cdots, \hat{a}_{n}$ of $\hat{B}=B(\hat{R}, 1)$ form a basis of $\hat{R}$.

Let $\hat{\mu}(\hat{a})=\mu(a)$ by definition. Since we have not proved that $\mu$ is a continuous linear functional on $R$, we cannot use Theorem 4.3 to establish that $\hat{\mu}$ is a linear functional on $\hat{R}$. We can however show $\hat{\mu}$ has these properties by a slightly different method. First, $\hat{\mu}$ is well defined. Indeed, if $a, b \in \hat{a}$, then $|a-b| \in \hat{0}$ and hence $\mu\left(\bar{e}_{|a-b|}\right)=0$. Since $|a-b| \bar{e}_{|a-b|}=|a-b|$, it follows by the Cauchy inequality that

$$
0 \leqq \mu(|a-b|) \leqq\left(\mu\left(|a-b|^{2}\right)\right)^{1 / 2}\left(\mu\left(\bar{e}_{|a-b|}\right)\right)^{1 / 2}=0
$$

and hence $\mu(a)=\mu(b)$. The linearity of $\hat{\mu}$ is a direct consequence of the result that $U_{\mu}$ is an ideal.

Since the atoms $\hat{a}_{1}, \cdots, \hat{a}_{n}$ of $\hat{B}$ form a basis of $\hat{R}$ every element $\hat{a}$ of $\hat{R}$ can be written

$$
a=\sum_{k=1}^{n} \alpha_{k} \hat{a}_{k}
$$

and

$$
\hat{\mu}(\hat{a})=\sum_{k=1}^{n} \alpha_{k} \hat{\mu}\left(\hat{a}_{k}\right) .
$$

If we define $\phi_{k}(a)=\alpha_{k}$, then it is clear that each $\phi_{k}$ is a ring-homomorphism of $\hat{R}$ onto the real field. Thus $\hat{\boldsymbol{\mu}}(\hat{a})$ is a finite linear combination of ring-homomorphisms:

$$
\hat{\mu}(a)=\sum_{k=1}^{n} \phi_{k}(a) \hat{\mu}\left(a_{k}\right) .
$$

Let $\Phi_{k}(a)=\phi_{k}(\hat{a})$ where $a \in \hat{a}$. Then $\Phi_{k}$ is a ring-homomorphism of $R$ onto the real field and by the definition of $\boldsymbol{\mu}$,

$$
\mu(a)=\hat{\mu}(\hat{a})=\sum_{k=1}^{n} \hat{\mu}\left(\hat{d}_{k}\right) \Phi_{k}(a) .
$$


Therefore $\mu$ is a finite linear combination of ring-homomorphisms and the proof is complete.

The following theorem is an important result in the theory of $F$-rings. A proof appears elsewhere [4] but the present proof is included because of its simplicity.

\section{TheOREM 5.5. If $M$ is a real maximum ideal of $R$, then $M$ is closed.}

Proof. Let $a(M)$ designate the real number associated with $a \in R$ under the homomorphism of $R$ onto $R-M$. Then $\mu(a)=a(M)$ is a non-negative linear functional on $R$. By Lemma 5.2, $\mu$ is a countably additive two-valued measure on $B=B(R, 1)$. The ideal $M \cap B$ of $B$ is therefore closed, and hence [4, Lemma 2] the ideal $M$ is also closed.

From [2, Theorem 5] it follows that if $R$ is a regular $F$-ring, then a maximal ideal of $R$ is closed if and only if it is real. The following corollary is a direct consequence of this remark and Theorem 5.4.

Corollary 5.6. If $B(R, 1)$ contains no closed maximal ideals, then there are no bounded linear functionals defined on $R$.

It is possible to provide a large class of examples of regular $F$-rings which have only the trivial bounded linear functional defined on them. A measurable space $(\Omega, \mathfrak{F})$ is said to have property $(U)$ if every nontrivial countably additive two-valued measure $\nu$ on the $\sigma$-algebra $\mathfrak{F}$ is fixed, that is, there exists a point $p \in \Omega$ such that $\nu(A)=1$ if and only if $p \in A$. Examples of measurable spaces with property $(U)$ are:

(i) Ulam spaces, that is, those where $|\Omega|$ is nonmeasurable and $\mathfrak{F}$ is the set of all subsets of $\Omega$.

(ii) The Lebesgue measurable space, that is the space where $\Omega$ designates the unit interval and $\mathfrak{F}$ the collection of all Lebesgue measurable subets of $\Omega$. That the Lebesgue measurable space has property $(U)$ follows from the nested intervals theorem.

(iii) The measurable space where $\Omega$ is both a $P$-space and a $Q$-space [5] and $\mathfrak{F}$ is the $\sigma$-algebra of all open-closed subsets of $\Omega$. This space has property $(U)$ by Theorem 5.5 and [2, Theorem 5 ].

If $(\Omega, \mathfrak{F})$ is a measurable space with property $(U)$ and $\mathfrak{A}$ is a closed ideal of $\mathfrak{F}$ with the property that the union of its elements is $\Omega$, then the quotient Boolean algebra $\mathfrak{F} / \mathfrak{A}$ is $\sigma$-complete. The assumption of the existence of a closed maximal ideal of $\mathfrak{F} / \mathfrak{A}$ implies the existence of a closed maximal ideal $\mathfrak{M}$ of $\mathfrak{F}$ with the property that the union of the elements of $\mathfrak{M}$ is $\Omega$, and this then implies that there exists a nonfixed countably additive two-valued measure on $\mathfrak{F}$. Let $R$ be a regular $F$-ring for which $B(R, 1)$ is isomorphic to $\mathfrak{F} / \mathfrak{A}$. From [3] it follows that $R$ is unique up to an isomorphism and (Corollary 5.6) there are no nontrivial bounded linear functionals on $R$. 
The following theorem is a direct consequence of the above remarks.

Theorem 5.7. Let $(\Omega, \mathfrak{F})$ be a measurable space with property $(U)$ and let $\mathfrak{A}$ be a closed ideal of $\mathfrak{F}$ such that $\cup \mathfrak{A}=\Omega$. If $R$ is a regular $F$-ring with identity 1 such that $B(R, 1)$ is isomorphic to the quotient $\sigma$-complete Boolean algebra $\mathfrak{F} / \mathfrak{A}$, then $R$ possesses only the trivial bounded linear functional.

A characterization of those linear functionals on an $F$-space $L$ with weak order unit 1 which can be extended to bounded linear functionals on $R(L, 1)$ can now be given. Let $\Omega^{\prime}$ stand for the class of closed maximal $l$-ideals of $L$ and let $F\left(\Omega^{\prime}\right)$ stand for the class of finite linear combinations of linear functionals $\gamma_{M}$ on $L$ with kernels $M$ in $\Omega^{\prime}$. It is a simple matter to verify that $F\left(\Omega^{\prime}\right)$ forms a complete vector lattice under the order relation: $\sum_{i=1}^{n} \alpha_{i} \gamma_{M_{i}} \geqq 0$ if and only if $\alpha_{i} \geqq 0$ for $1 \leqq i \leqq n$.

Theorem 5.8. A linear functional $\mu$ on $L$ can be extended to a bounded linear functional on $R(L, 1)$ if and only if $\mu \in F\left(\Omega^{\prime}\right)$.

Proof. If $\mu \in F\left(\Omega^{\prime}\right)$, then $\mu=\sum_{i=1}^{n} \alpha_{i} \gamma_{M_{i}}$ where $M_{i} \in \Omega^{\prime}$. By Theorem 3.3 there is a one-to-one mapping $\phi: S \rightarrow S \cap L$ of the class of closed maximal ideals of $R(L, 1)$ onto $\Omega^{\prime}$. From Theorem 4.2 it follows that $\gamma_{M_{i}}(1) \neq 0$ for all $1 \leqq i \leqq n$ because $\gamma_{M_{i}}(1)=0$ implies $\gamma_{M_{i}}(|f|)=0$ for all $f \in L$. Let $\mu^{\star}$ be the bounded linear functional on $R(L, 1)$ which has the form:

$$
\mu \star=\sum_{i=1}^{n} \alpha_{i} \cdot \gamma_{M_{i}}(1) \cdot \gamma_{\phi^{-1}\left(M_{i}\right)}^{\star}
$$

where $\gamma_{\phi}^{\star-1}{ }_{\left(M_{i}\right)}$ designates the ring-homomorphism associated with the closed (real) maximal ideal $\phi^{-1}\left(M_{i}\right)$ of $R(L, 1)$.

The restriction of $\mu^{\star}$ to $L$ is the functional $\mu$. To show this, note that for $x, y \in L, x-y \in M_{i}$ if and only if $x-y \in \phi^{-1}\left(M_{i}\right)$ and that if $\gamma_{i}^{\star}$ is used to designate $\gamma_{\phi}^{\star-1}\left(M_{i}\right)$, then $x-\gamma_{i}^{\star}(x) \cdot 1 \in \phi^{-1}\left(M_{i}\right)$. Here again $L$ is identified with its isomorphic copy in $R(L, 1)$. Since $\gamma_{i}^{\star}(x) \cdot 1 \in L$, we have

$$
x-\gamma_{i}^{\star}(x) \cdot 1 \in M_{i}
$$

and hence $\gamma_{M_{i}}(x)=\gamma_{i}^{\star}(x) \gamma_{M_{i}}^{\star}(1)$. Thus for $x \in L$,

$$
\mu \star(x)=\sum_{i=1}^{n} \alpha_{i} \gamma_{M_{i}}(x)=\mu(x) .
$$

Conversely if $\mu$ is a nontrivial functional on $L$ which has a bounded linear extention $\mu^{\star}$ to $R(L, 1)$, then there exists (Theorem 5.4) a set $\left\{M_{1}, M_{2}, \cdots, M_{n}\right\}$ of closed maximal ideals of $R(L, 1)$ such that

$$
\mu \star=\sum_{i=1}^{n} \alpha_{i} \gamma_{M_{i}}^{\star}
$$


Here $\gamma_{\boldsymbol{M}_{i}}^{\star}$ is the ring-homomorphism of $R(L, 1)$ associated with the closed ideal $M_{i}$. The restriction of $\gamma_{M_{i}}^{\star}$ to $L$ is of the form $\gamma_{\phi\left(M_{i}\right)}$ and hence $\mu=\sum_{i=1}^{n} \alpha_{i} \gamma_{\phi\left(M_{i}\right)}$. Therefore $\mu \in F\left(\Omega^{\prime}\right)$.

\section{REFERENCES}

1. G. Birkhoff, Lattice theory, New York, 1948.

2. B. Brainerd, On a class of lattice-ordered rings, Proc. Amer. Math. Soc. vol. 8 (1957) pp. 673-683.

3. - On a class of lattice-ordered rings II, Nederl. Akad. Wetensch Proc. Ser. A. vol. 60 (1957) pp. 541-547.

4. - F-rings of continuous functions I, Canad. J. Math. vol. 11 (1959) pp. 80-86.

5. L. Gillman and M. Henriksen, Concerning rings of continuous functions, Trans. Amer. Math. Soc. vol. 77 (1954) pp. 340-362.

6. G. W. Mackey, Equivalence of a problem in measure theory to a problem in the theory of vector lattices, Bull. Amer. Math. Soc. vol. 50 (1944) pp. 719-722.

7. H. Nakano, Modern spectral theory, Tokyo, 1950.

8. J. M. H. Olmsted, Lebesgue theory on a Boolean algebra, Trans. Amer. Math. Soc. vol. 51 (1942) pp. 164-193.

9. E. C. Smith, Jr. and A. Tarski, Higher degrees of distributivity and completeness in Boolean algebras, Trans. Amer. Math. Soc. vol. 84 (1957) pp. 230-257.

UNiversity OF WeSTERN ONTARIO, London, Ontario, Canada

UNIVERSITY OF TORONTO, Toronto, Ontario, Canada 